\title{
Classificação multicritério dos portos de contêineres no Brasil ${ }^{1}$
}

\author{
Angélica Faria Campanhão \\ Instituto Tecnológico e das Ciências Sociais Aplicadas e da Saúde (Isecensa) \\ Fernando Leste Nauck Fabiano Filho \\ Instituto Tecnológico e das Ciências Sociais Aplicadas e da Saúde (Isecensa) \\ Juliana Landim Gomes Siqueira \\ Instituto Tecnológico e das Ciências Sociais Aplicadas e da Saúde (Isecensa) \\ Rafaela Landim Gomes Siqueira
}

Instituto Tecnológico e das Ciências Sociais Aplicadas e da Saúde (Isecensa)

Henrique Rego Monteiro da Hora

Instituto Federal de Educação, Ciência e Tecnologia Fluminense (Iff)

Helder Gomes Costa

Departamento de Engenharia de Produção da Universidade Federal Fluminense (Uff)

Recebido: 14/01/2014 Versão revisada (entregue): 18/08/2014 Aprovado: 21/08/2014

\section{Resumo}

O atual cenário dos portos no Brasil é caracterizado por uma intensa competição pela conquista e manutenção de mercados consumidores. Especificamente, essas organizações estão buscando o aperfeiçoamento, não somente dos processos produtivos (bens e serviços), mas também em processos organizacionais e administrativos. Logo, destaca-se a importância, para a economia mundial, de portos eficientes, bem localizados e com capacidade suficiente para atender às necessidades de transporte e manutenção do mercado consumidor. Diante da busca por competitividade, o problema contemplado nesta pesquisa procura responder a questão de como está a situação dos portos brasileiros. O objetivo do trabalho é avaliar criticamente os portos no Brasil a partir de uma análise classificatória multicriterial. Foi utilizado o método ELECTRE TRI que se apoia em um sistema de preferência com subordinação. Para performance do estudo foram executadas as seguintes atividades: levantamento dos portos de contêineres brasileiros e de indicadores, identificação e seleção dos principais indicadores de infraestrutura e desempenho, atribuição de peso aos critérios e construção das matrizes do modelo. Através da

${ }^{1}$ Este trabalho é resultado de pesquisa realizada no âmbito de uma pós-graduação em Logística Portuária, na região de instalação do Super Porto do Açu. 
análise dos dados constatou-se que a situação dos portos é heterogênea, com alguns portos apresentando bom desempenho geral e muitos com desempenho regular e ruim.

Palavras-chave | Brasil; classificação de portos; ELECTRE TRI; portos de contêineres.

Código JEL | C30; 018; R48.

\title{
MULTICRITERIA RANKING OF THE CONTAINER PORTS IN BRAZIL
}

\begin{abstract}
Nowadays the scenario of the ports in Brazil is characterized by intense competition for the achievement and maintenance of consumer markets. Specifically, these organizations are seeking improvement, not only of production processes (goods and services) but also of organizational and administrative processes. Therefore, we highlight the importance for the world economy, to have efficient and well located ports, and with enough capacity to meet the transportation needs and maintaining the consumer market. Given this search for competitiveness, the problem presented by this research seeks to answer the question of how is the situation of Brazilian ports. According to the scenario presented the research objective is to assess and critically analyze the ports in Brazil, carrying out a multicriteria analysis to classify them. We applied the ELECTRE TRI method, which is based on a preference system with subordination. For the study performance the following activities were carried out: a survey of Brazilian container ports and indicators, identification and selection of key indicators of infrastructure and performance, different weights to the criteria and matrices construction of the model. Through data analysis, we found that the situation of the ports is heterogeneous, with some ports performing well overall, and many with fair and poor performance.
\end{abstract}

Keywords | Brazil; classification of ports; containers ports; ELECTRE TRI.

JEL-Code | C30; 018; R48.

\section{CLASIFICACIÓN MULTICRITERIO DE LOS PUERTOS DE CONTENEDORES EN BRASIL}

\section{Resumen}

Actualmente, el escenario de los puertos en Brasil se caracteriza por una intensa competencia por la conquista y conservación de los mercados de consumo. Específicamente, estas organizaciones están buscando mejorar no sólo los procesos de producción (bienes y servicios), sino también los procesos organizacionales y administrativos. Por lo tanto, ganan importancia para la economía mundial, los puertos eficientes, bien situados y con capacidad suficiente para satisfacer las necesidades de transporte y manutención del mercado consumidor. Frente a esta búsqueda de competitividad, esta investigación busca responder a la pregunta de cómo está la situación de los puertos brasileños. El objetivo del trabajo es hacer una evaluación crítica de los puertos en Brasil a partir de un análisis clasificatoria multicriterial. El método utilizado fue el ELECTRE TRI que se apoya en un sistema de preferencia con subordinación. Para aplicar este método, se realizaron las siguientes actividades: levantamiento de los puertos brasileños de contenedores y de indicadores, identificación y selección de los principales indicadores de infraestructura y desempeño, atribución de peso a los criterios y construcción de las matrices del modelo. A través del análisis de los datos se constató que la situación de los puertos es heterogénea, con algunos puertos que muestran un buen rendimiento general y muchos con desempeño regular y malo. 
Palabras-clave | Brasil; clasificación de puertos; ELECTRE TRI; puertos de contenedores.

Código JEL | C30; 018; R48.

\section{Introdução}

O sistema portuário no Brasil originou-se no período de colonização do país, época em que a maioria das mercadorias comercializadas entre os países e as localidades internas, como também os imigrantes vindos da Europa, eram transportados através do transporte aquaviário. Logo, o setor portuário possui relevante importância para a economia, principalmente em relação ao comércio internacional (ROCHA; MORATO, 2009).

Após o setor rodoviário, o setor portuário no Brasil se destaca como o segundo mais importante quando se trata dos transportes nacionais. Segundo estudo realizado por Lacerda (2005), cerca de 75\% do valor do comércio exterior brasileiro são movimentados através dos portos nacionais. O Brasil possui um dos litorais mais extensos do mundo, porém é criticado devido à ineficiência apresentada em seus serviços e administração. Essa situação prejudica consideravelmente a participação do país nos mercados mundiais.

Mesmo sendo consideradas algumas limitações na área portuária, Kirchner e Medeiros (2010) afirmam que o Brasil está se desenvolvendo em diversas áreas depois do auge da crise econômica mundial, em 2009, principalmente na quantidade de mercadorias que entra e sai do país através dos portos. Os dados do boletim portuário do segundo trimestre mostram que nos portos organizados e nos terminais de uso privativo a movimentação de carga foi de 182 milhões de toneladas, apresentando uma taxa de crescimento de 9,6\% frente ao mesmo período de 2009. Perante as expectativas relacionadas à economia brasileira e dos dados disponíveis referentes ao primeiro trimestre de 2010, foi estabelecida uma previsão de movimentar cerca de 760 milhões de toneladas, que representa um aumento de 3,8\% em relação ao ano anterior, quando se movimentou um total de 732,9 milhões. Mesmo sendo considerada uma estimativa otimista, será muito difícil os números atingirem os patamares registrados em 2008, quando foi movimentado 768,3 milhões.

Em dados do primeiro trimestre de 2014 (ANTAQ, 2014), observa-se um crescimento de 4,6\% nas movimentações em relação ao mesmo período do ano anterior, apontando para a recuperação do setor, que se apresenta em franco crescimento. Especificamente sobre os portos de contêineres, objeto deste estudo, o incremento alcançado foi de $10 \%$ na comparação entre períodos, alcançando a marca de 22,6 milhões de toneladas. 
Com uma costa de 8,5 mil quilômetros navegáveis, o Brasil possui um setor portuário que movimenta anualmente cerca de 700 milhões de toneladas das mais diversas mercadorias e responde, sozinho, por mais de $90 \%$ das exportações. Para o transporte de cargas no Brasil o modal aquaviário possui um dos menores custos, perdendo apenas para o transporte aéreo e dutoviário, de acordo com estudos desenvolvidos pela Coppead. O sistema portuário brasileiro é composto por 37 portos públicos, entre marítimos e fluviais. Desse total, 18 são delegados, concedidos ou tem sua operação autorizada à administração por parte dos governos estaduais e municipais. Existem ainda 42 terminais de uso privativo e três complexos portuários que operam sob concessão à iniciativa privada (KIRCHNER; MEDEIROS, 2010).

Atualmente, o cenário apresentado é caracterizado por uma intensa competição pela conquista e manutenção de mercados consumidores, sendo que as organizações privadas e públicas vêm buscando a obtenção da vantagem competitiva das mais variadas formas. Essas organizações estão buscando o aperfeiçoamento dos processos produtivos (bens e serviços), organizacionais e administrativos. Neste aspecto, a competitividade encontra-se fundamentada no trinômio qualidade, produtividade e flexibilidade (FREITAS; COSTA, 2003).

O problema contemplado pela pesquisa procura responder à questão de como está a situação dos portos brasileiros. Adota-se como hipótese que a situação é heterogênea, com alguns portos apresentando bom desempenho geral, e muitos com desempenho regular e ruim. O objetivo é avaliar e analisar criticamente os portos no Brasil, a partir de uma análise classificatória multicriterial.

\section{Abordagem metodológica}

\section{Classificação da pesquisa}

Do ponto de vista de sua natureza, a pesquisa é considerada aplicada, pois se utiliza de técnicas já desenvolvidas pela ciência para resolver soluções de interesse locais por meio de práticas consagradas (SILVA; MENEZES, 2005). Com base em seus objetivos, este estudo caracteriza-se como descritivo, pois visa descrever as características de determinada população ou fenômeno. Envolve o uso de técnicas padronizadas de coleta de dados e assume, em geral, a forma de levantamento (GIL, 2007).

Quanto à abordagem do problema, considera-se a abordagem qualitativa na avaliação dos itens na escala nominal, mas quantitativa no uso de técnicas algébricas para determinação dos resultados (SILVA; MENEZES, 2005). 
Quanto aos procedimentos técnicos, lista-se a pesquisa documental nos catálogos de indicadores do sistema portuário; pesquisa bibliográfica do arcabouço teórico de sustentação do trabalho; estudo de caso para tentar conhecer em profundidade o a realidade estudada; e levantamento para ponderação dos critérios (SILVA; MENEZES, 2005).

\section{Procedimentos técnicos}

A pesquisa documental foi realizada na lista de indicadores disponibilizados para mensurar a infraestrutura e o desempenho dos portos, no portal do Sistema Portuário Nacional (SEP, 2014) da Agência Nacional de Transportes Aquaviários (ANTAQ), que atua como órgão de regulação e supervisão dos portos organizados e terminais privativos, sendo assim uma fonte segura e válida de informações para a problemática (MONTEIRO; HORA, 2014).

Para classificação dos portos, segundo os seus desempenhos, é utilizado o método ELECTRE TRI (FIGUEIRA; MOUSSEAU; ROY, 2005), descrito adiante, que congrega todos os diferentes critérios, com suas diferentes ponderações, atribuindo cada alternativa (porto) a uma classificação pré-determinada. O método ou prevê dois resultados, um otimista e um pessimista. Para a análise dos resultados, adota-se uma postura pessimista, por se tratar de uma análise de serviços públicos que demandam melhorias constantes.

A atribuição de importância para cada critério é realizada por consulta a especialistas, sendo cinco profissionais com especialização na área portuária ou atuação profissional na área. A técnica dos cem pontos foi escolhida, de modo que cada especialista distribuísse essa centena por todos os critérios. A média aritmética simples dos pesos atribuídos para cada critério é considerada como o peso dele próprio. A técnica de consulta a especialista é considerada válida e confiável (HORA; MONTEIRO; ARICA, 2010).

\section{Corte temporal, população e amostra}

A crise econômica de 2008 no setor imobiliário americano provocou uma onda de recessão na maioria dos países capitalistas, ganhando proporções mundiais, sendo que até os dias atuais esse cenário não voltou à normalidade (MOTA, 2013). Diante deste cenário, opta-se por fazer este estudo com dados do ano de 2007, que pode ser considerado o último ano consolidado, em que os portos brasileiros trabalharam em condições econômicas plenas, não tendo seu desempenho afetado pelo cenário mundial. 
Apesar dos dados de 2007 causarem a impressão de serem desatualizados, a realidade portuária demanda grandes investimentos de infraestrutura e não sofre mudanças drásticas em curto período de tempo. Assim sendo, as análises com o uso dos dados de 2007 são plenamente válidas para os dias atuais, pois aferem o desempenho tendo por objeto praticamente a mesma infraestrutura atual.

\section{População e amostra}

O delineamento desta pesquisa cerca o universo dos portos brasileiros. Dado seu caráter público e a disponibilidade de informações, a amostra adotada é delineada pelas informações disponíveis no portal governamental para gestão dos portos.

O cenário brasileiro à época do corte temporal contava com 79 portos, sendo 37 sob gestão pública e 42 sob gestão privada, além de três complexos portuários que operam sob concessão à iniciativa privada (ANTAQ, 2013).

O estudo se detém na amostra de 19 portos e 37 terminais, de onde se extraem 12 portos brasileiros com dados no Sistema Portuário Nacional (SEP, 2014), utilizando técnica amostral não-probabilística intencional, tendo como principal critério a disponibilidade de dados (SILVA; MENEZES, 2005).

De acordo com Kirchner e Medeiros (2010), os portos podem ser definidos segundo a tipagem de sua carga, podendo ser classificados como de carga conteinerizada, granel sólido, granel líquido e de carga geral solta.

O estudo adota os portos de contêineres para o estudo de caso. Entende-se por contêiner um recipiente construído de material resistente, destinado a propiciar o transporte de mercadorias com rapidez, segurança e inviolabilidade, dotado de dispositivo de segurança aduaneira, devendo atender às condições técnicas e de segurança previstas pela legislação nacional e pelas convenções internacionais ratificadas pelo Brasil (BRASIL, 1977).

\section{Decisão multicriterial}

Pode-se considerar que existem atualmente duas grandes escolas de estudo dos métodos multicritério: A Escola Americana e a Escola Francesa. A origem dessas Escolas e a técnica destacada por cada uma delas remontam aos primeiros trabalhos cientificamente formalizados e aceitos (GOMES, 2007; MOREIRA, 2007). As ações como também a técnica que irão ser utilizadas são distintas ao longo do processo de decisão.

A Escola Francesa ou Europeia permite uma modelagem mais flexível do problema, pois não admite necessariamente a comparabilidade entre todas as 
alternativas. Segundo Gomes; González-Araya; Carignano (2004) uma alternativa pode ser preferida a outra de modo estrito ou fraco, pode ser indiferente, mas também pode ser incomparável, o que não é admitido na Escola Americana.

Segundo Parreiras (2006), os métodos de decisão da Escola Francesa caracterizamse por apresentar dois estágios. No primeiro, são realizadas comparações entre cada alternativa pertencente a $A$ e as demais. A partir dessas comparações são definidas relações de sobreclassificação entre cada par de alternativas de tal maneira que, dadas $a, b \in A$, serão pelo menos tão boas quanto $b$, então se pode dizer que $a$ sobreclassifica $b$. No segundo estágio, essas relações são exploradas por meio de um conjunto de diretrizes, tendo como objetivo ordenar as alternativas da melhor para a pior, classificar as alternativas em categorias predefinidas ou obter a melhor alternativa de $A$.

\section{Sistema de preferências nos métodos ELECTRE}

O modelo de preferência imposto ao agente de decisão não considera completamente a posição deste em relação a duas alternativas. Se o agente de decisão expressar sua preferência entre duas soluções admissíveis, não se pode garantir que as suas informações sejam precisas e completas (GOICOECHEA; HANSEN; DUCKSTEIN, 1982). O decisor pode deparar-se com uma das quatro situações ao comparar duas alternativas (ALMEIDA, 2013; OLIVEIRA, 2003):

a) uma alternativa é preferida à outra com preferência forte, também denominada preferência sem hesitação;

b) uma alternativa é preferida à outra com preferência fraca, também denominada preferência com hesitação;

c) uma alternativa é indiferente à outra;

d) uma alternativa é incomparável à outra.

Diante dessa situação, segundo Gomes; González-Araya; Carignano (2004) é necessário incrementar o sistema de preferências com mais duas situações: a incomparabilidade e a preferência fraca, compondo o Sistema Fundamental de Relações de Preferências.

\section{Transitividade nas relações de preferência}

Conceito importante e que deve ser reforçado, que contrapõe a visão da Teoria Clássica e que também é utilizado pela Escola Francesa, refere-se à transitividade das relações de Preferência Estrita e Indiferença. Moreira (2007) define que, de 
acordo com a Teoria Clássica, se $a I_{c}$ e $b I_{c}=>a I c$; e $a \mathrm{~Pb}$ e bPc $=>a P c$, o que na prática observamos não se tratar de uma regra rígida, uma vez que o decisor pode sim vir a apresentar, por exemplo, preferências do tipo $a Q b$ e $b Q_{c}=>a P c$.

Gomes; González-Araya; Carignano (2004) mostram excelente exemplo para ilustrar esse tema. No que se refere à indiferença, existe o clássico exemplo das xícaras de café dispostas em uma fila, de modo que seja adicionado um pouco de açúcar a partir da primeira xícara. Ao longo da fila, aumenta-se a quantidade de açúcar por xícara, sem que o degustador perceba a diferença do sabor adocicado entre xícaras adjacentes. Aquelas que estão situadas nos extremos da fila, parece óbvio, serão indiferentes no sabor, sendo assim, quanto maior for a distancia entre as xícaras na fila, mais perceptível a mudança do paladar adocicado.

\section{Psendocritérios}

Com o objetivo de reduzir a imprecisão e a incerteza que se estabelece nesses momentos de definição de critérios, utiliza-se a delimitação de limites de tolerância para a passagem de uma situação de preferência para outra. Esses critérios que possuem limites de preferência e indiferença foram denominados pseudocritérios (ALMEIDA, 2013; GOMES; GOMES, 2012; GOMES; GONZÁLEZ-ARAYA; CARIGNANO, 2004).

Moreira (2007) afirma que, novamente, de forma diversa do que a Teoria Clássica aceitava, uma diferença positiva entre duas alternativas 'a' e 'b', dado um critério $g(a)-g(b)$ não deverá ser interpretada diretamente como sendo uma preferência pela opção 'a'. Desta forma, esses limites podem ser representados da seguinte forma (ALMEIDA, 2013; GOMES; GOMES, 2012; GOMES; GONZÁLEZ-ARAYA; CARIGNANO, 2004):

- Limite de preferência (p):

$$
\begin{aligned}
& \mathrm{aPb} \Leftrightarrow \mathrm{g}(\mathrm{a})-\mathrm{g}(\mathrm{b})>+\mathrm{p} \\
& \mathrm{aIb} \Leftrightarrow-\mathrm{q}=\mathrm{g}(\mathrm{a})-\mathrm{g}(\mathrm{b})=+\mathrm{q} \\
& \mathrm{aQb} \Leftrightarrow \mathrm{q}<\mathrm{g}(\mathrm{a})-\mathrm{g}(\mathrm{b})<\mathrm{p}
\end{aligned}
$$

- Limite de indiferença (q):

- Situação de preferência fraca:

\section{O método ELECTRE TRI}

O método ELECTRE TRI pertence à família ELECTRE (um dos primeiros métodos da Escola Francesa de Apoio Multicritério à Decisão, que propôs uma série de métodos a partir do mesmo sistema de preferência). Este método considera a problemática tipo beta (P.ß), que classifica as diversas alternativas para a solução de um problema por meio da comparação de cada alternativa potencial 
com uma referência estável (padrão/alternativa de referência) (DOUMPOS; ZOPOUNIDIS, 2002; ROY; BOUYSSOU, 1993).

Para haver essa comparação, são determinados os pesos $\left(k_{1}, k_{2}, k_{3}, k_{4}\right.$, $k_{5}, \ldots, k_{j}$ ), onde $k_{j}$ é o peso do critério j. É importante destacar que o somatório dos coeficientes dos critérios deve ser igual a 1. Para evitar o uso de uma escala de conversão, devem ser atribuídos valores entre 0 e 1 para os coeficientes dos critérios (LIMA; ALMEIDA; ALMEIDA, 2010).

Desta forma, atribui as alternativas a categorias pré-definidas e esse processo de atribuição de uma alternativa resulta da comparação da mesma com os valores padrões que definem os limites (bordas) superiores e inferiores das categorias (ALMEIDA, 2013; ALMEIDA-DIAS; FIGUEIRA; ROY, 2010).

Figura 1 - Ilustração de uma alternativa ' $a_{i}$ ' tendo suas avaliações ' $g_{j}$ ' sendo alocada nas classes ' $\mathrm{C}_{\mathrm{h}}$ '

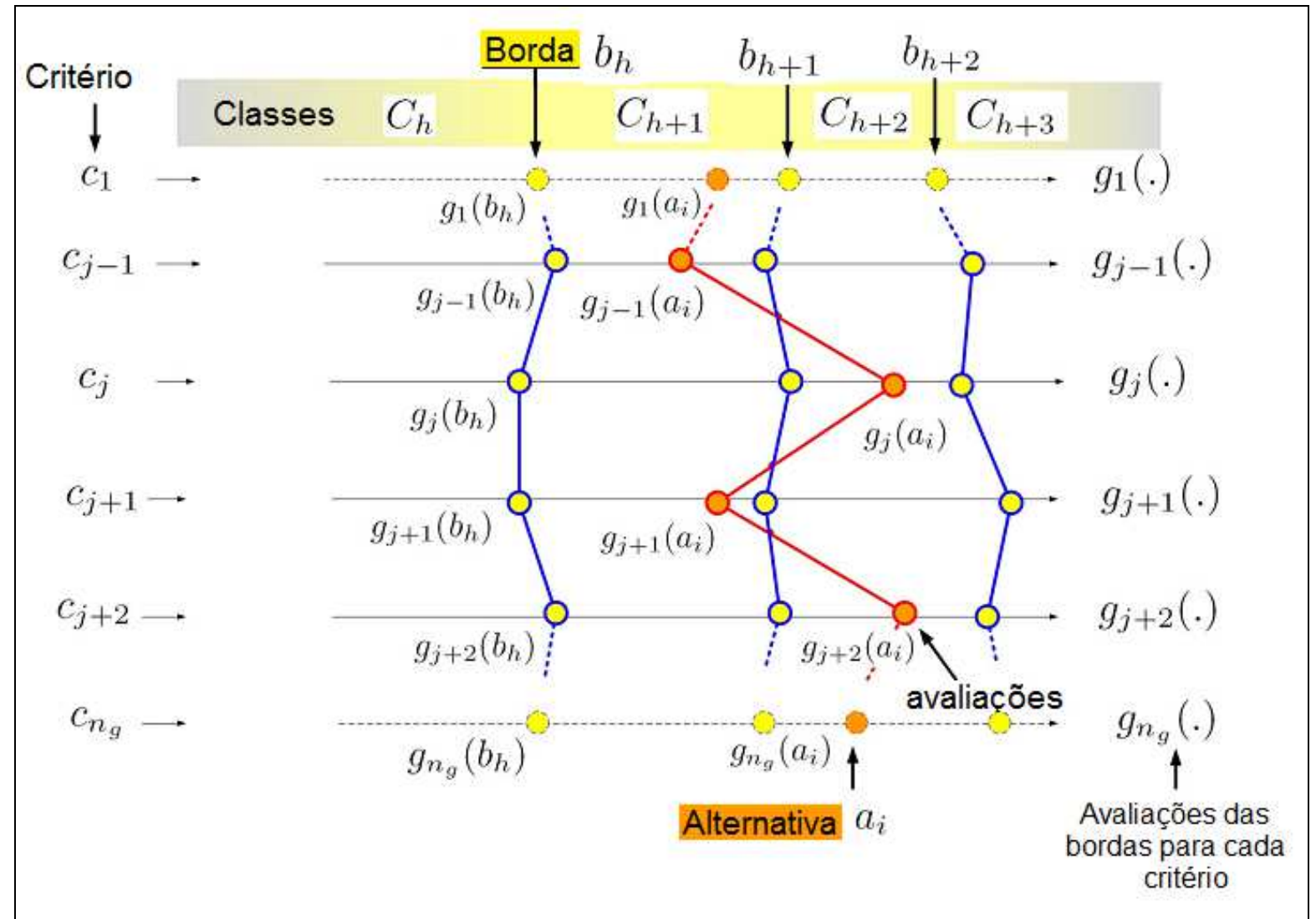

Fonte: Dezert; Tacnet (2012).

O ELECTRE TRI é um método de sobreclassificação, também denominado método de subordinação (termo em inglês: outranking), e fundamenta-se na 
construção de uma relação de sobreclassificação que incorpora as preferências estabelecidas pelo decisor diante dos problemas e das alternativas disponíveis (HORA et al., 2008; SZAJUBOK; MOTA; ALMEIDA, 2006).

A relação de sobreclassificação S é uma relação binária definida em A tal que aSb se a é pelo menos tão boa quanto b (ROY; BOUYSSOU, 1993). Sendo conhecidas as alternativas ditas como referência, $\mathrm{b}_{0}, \mathrm{~b}_{1}, \mathrm{~b}_{2}, \ldots, \mathrm{b}_{\mathrm{n}}$, e os critérios $\mathrm{i}_{1}, \mathrm{i}_{2}, \ldots, \mathrm{i}_{\mathrm{n}}$, definem-se as categorias $\mathrm{E}^{1}, \mathrm{E}^{2}, \ldots, \mathrm{E}^{\mathrm{m}}$. Assim, para um dado critério $\mathrm{i}$, a alternativa a será localizada em uma determinada categoria, em função de sua avaliação $g_{i}$ (a) (COSTA et al., 2007; GOMES; GONZÁLEZ-ARAYA; CARIGNANO, 2004).

Fundamentado na afirmação de $a S b$ ou $a S b$, deve-se verificar segundo (COSTA; SOARES; OLIVEIRA, 2004; ROY; BOUYSSOU, 1993):

- Concordância: para que $a S b$ (ou $b_{b} S a$ ) seja a condição aceita, uma maioria suficiente de critérios deve ser a favor desta afirmação; e

- Não-discordância: quando na condição de sobreclassificação de concordância esperada, nenhum dos critérios na minoria deve opor-se à afirmação $a S b_{b}$ (ou $\left.b_{b} S a\right)$.

De acordo com os princípios de concordância e não-discordância deve-se construir um índice $\sigma(a, b h)$ que representa o grau de credibilidade de uma relação de subordinação $\mathrm{S}$, seguindo as seguintes etapas, como sugeriu (DEZERT; TACNET, 2013; FIGUEIRA; MOUSSEAU; ROY, 2005).

(i) cálculo do índice de concordância parcial $c_{j}\left(a, b_{b}\right)$ e $c_{j}\left(b_{b}, a\right)$;

(ii) cálculo do índice de concordância global $c\left(a, b_{b}\right)$;

(iii) cálculo dos índices de discordância $d_{j}\left(a, b_{b}\right)$ e $d_{j}\left(b_{b}, a\right)$.

(iv) cálculo do índice de credibilidade $\sigma\left(a, b_{b}\right)$ da relação de subordinação.

Segundo Costa (2004), após o cálculo do índice $\sigma\left(a, b_{b}\right)$, utiliza-se um nível de corte- $\lambda$, que é considerado como o menor valor do índice de credibilidade compatível com a afirmação de que " $a$ subordina $b_{b}$ ".

Em seguida, são atribuídos dois procedimentos de alternativas: o procedimento pessimista e o otimista, como sugerem (DOUMPOS; ZOPOUNIDIS, 2002; FREITAS; COSTA, 2000).

No procedimento pessimista, compara-se a alternativa $a$ sucessivamente a $b_{i}$, para $i=p, p-1, \ldots, 0$. Sendo $b_{b}$ o primeiro valor limite tal que $a \mathrm{~S} b_{b}$, atribuir $a$ à categoria $C_{b+1}\left(a-C_{b+1}\right)$. Se $b_{b-1}$ e $b_{b}$ denotam os valores do limite inferior e superior da categoria $C_{b}$, este procedimento atribui a alternativa $a$ à mais alta categoria $C_{b}$ tal que $a$ subordina o valor $b_{b-1}$, isto é, $a S b_{b-1}$.

No procedimento otimista compara-se a alternativa $a$ sucessivamente a $b_{i}, i=1$, $2, \ldots, p$. Sendo $b_{b}$ o valor limite tal que $b_{b} \mathrm{P} a$, atribuir $a$ à categoria $C_{b}\left(a-C_{b}\right)$. Este 
procedimento atribui $a$ à categoria $C_{b}$ mais inferior, para a qual o valor do limite superior $b_{b}$ é preferido a $a$, isto é, $b_{b} \mathrm{P} a$.

\section{Estudo de Caso}

\section{Alternativas consideradas}

Os portos considerados no estudo são delimitados pela característica de movimentação de contêineres, que se aceita serem os principais do país, representando satisfatoriamente a realidade brasileira. São descritos resumidamente a seguir (ANTAQ, 2013; SEP, 2014):

- (A1) - Porto de Manaus: O Porto de Manaus, inaugurado em 1907, é considerado o mais original do Brasil. Construído em um cais flutuante, acompanha o nível das águas do rio Negro, em épocas de grande cheias. O porto está situado entre a praia de São Vicente e a rampa do Mercado Municipal Adolpho Lisboa;

- (A2) - Porto de Belém: O Porto de Belém é um porto brasileiro localizado na cidade de Belém, no estado do Pará. Caracteriza-se pela exportação de castanha, madeira e bauxita;

- (A3) - Porto de Vila do Conde: O porto de Vila do Conde foi inaugurado em 24 de outubro de 1985, como resultado dos acordos de cooperação econômica firmados entre o Brasil e o Japão em setembro de 1976, nos quais o governo brasileiro assumiu a responsabilidade pela implantação da infraestrutura portuária, rodoviária e urbana, para o escoamento de alumínio produzido no complexo industrial da Albrás/Alunorte (PA);

- (A4) - Porto de Fortaleza: É um porto da costa do Nordeste brasileiro, localizado no estado do Ceará, na cidade de Fortaleza. O Porto de Fortaleza é um dos principais portos da navegação de cabotagem do Brasil em movimentação de cargas;

- (A5) - Porto de Suape: O Porto de Suape é um porto brasileiro localizado no estado de Pernambuco, entre os municípios de Ipojuca e Cabo de Santo Agostinho, mais exatamente na Foz do Rio Ipojuca;

- (A6) - Porto de Salvador: O Porto de Salvador é um porto brasileiro localizado na cidade de Salvador, capital do estado da Bahia. É situado numa área naturalmente protegida, a Baía de Todos os Santos;

- (A7) - Porto de Vitória: O Porto de Vitória é o principal terminal de transporte marítimo da cidade de Vitória, capital do Espírito Santo. Possui um 
sistema de transporte intermodal bastante atrativo e competitivo, com uma estrutura de transporte rodoviário, ferroviário e marítimo a bons níveis;

- (A8) - Porto de Santos: O Porto de Santos, localizado no município de Santos, no estado de São Paulo, é o principal porto brasileiro. É também o porto de contêineres mais movimentado da América Latina. Ele apresenta grande diversidade de terminais de movimentação de cargas - granéis sólidos e líquidos e carga geral;

- (A9) - Porto de São Francisco do Sul: É um porto brasileiro localizado na baía da Babitonga em São Francisco do Sul, no estado de Santa Catarina. É administrado pelo governo do estado de Santa Catarina, por meio da Administração do Porto de São Francisco do Sul (APSFS);

- (A10) - Porto de Itajaí: É um porto brasileiro localizado na cidade de Itajaí, no estado de Santa Catarina. É o principal porto de Santa Catarina, sendo o segundo maior do país em movimentação de contêineres. Os principais produtos exportados são madeira, pisos cerâmicos, máquinas, açúcar, papel e fumo, e os principais produtos importados são trigo, produtos químicos, motores, têxteis, papel e pisos cerâmicos;

- (A11) - Porto de Imbituba: Está localizado numa enseada aberta, junto à ponta de Imbituba, no litoral sul do estado brasileiro de Santa Catarina. O porto é controlado pela Companhia Docas de Imbituba S/A;

- (A12) - Porto de Rio Grande: É um porto brasileiro que está localizado na margem direita do canal do norte, que liga a Lagoa dos Patos ao Oceano Atlântico, no município gaúcho de Rio Grande, no estado do Rio Grande do Sul.

\section{Critérios utilizados}

Os critérios eleitos foram divididos em dois grandes grupos. O primeiro para caracterizar a infraestrutura que define a capacidade instalada, o segundo para caracterizar a capacidade de desempenho de cada porto (ANTAQ, 2013). Considera-se ainda a movimentação total de itens no ano base de 2007 (último dado disponível antes da crise mundial de 2008).

Os indicadores de infraestrutura são:

- Área (CR01): Compreende a área terrestre total ocupada pelo porto expresso em metros quadrados;

- Extensão da Costa (CR02): Representa a extensão do porto em contato com a área marítima, sendo a unidade de medida expressa em quilômetros; 
- Profundidade máxima (CR03): Representa a profundidade do porto, o calado máximo de operação, no canal de navegação. Como se sabe, é a profundidade do porto que já vem determinando em boa parte a rota dos navios;

- Quantidade de armazéns (CR04): Corresponde a quantidade total de armazéns dos portos. Os armazéns representam as áreas cobertas para armazenagem dos diversos tipos de cargas;

- Quantidade de berços (CR05): Esse indicador representa a quantidade total de berços de cada porto, sendo os berços uma instalação de acostagem, por onde ocorrem as movimentações das cargas.

Os indicadores de desempenho são:

- Frequência de Navios (CR06): Medida em números inteiros para identificar a quantidade de navios que estiveram no porto no presente período;

- Atendimento ao tráfego (CR07): Medido em percentagem da movimentação de carga através de cada terminal ou conjunto de berços em relação à movimentação total da carga ou mercadoria no porto (carga geral solta, granéis sólidos, granéis líquidos, contêineres e roll on roll off) ou por mercadoria principal;

- Consignação Média (CR8): Medida em toneladas por navio para a carga geral, granéis líquidos, roll on roll off e granéis sólidos e em unidades carregadas e descarregadas por navio, para contêineres: Indica a característica do tamanho de navio que frequenta o porto, para cada tipo de carga ou mercadoria, em cada terminal ou conjunto de berço;

- Prancha Média (CR9): Medida em unidades por hora, por navio, para contêineres e em toneladas por dia, por navio, para carga geral, roll on roll off, granéis líquidos e granéis sólidos, em cada terminal ou conjunto de berços. Indica a produtividade média de cada terminal ou conjunto de berços, medida em relação ao tempo de atracação dos navios, tomado como tempo de atendimento;

- Tempo Médio de espera (CR10): Indicador da qualidade do atendimento, em termos do tempo, medido em horas e minutos, gasto em espera de atracação dos navios de cada grupo de carga ou produto, para cada terminal ou conjunto de berços;

- Movimentação de Contêineres (CR11): Este indicador representa a quantidade total de carga movimentada em Peso (toneladas) por cada porto, através de contêineres. Os contêineres são recipientes de grandes dimensões destinados ao acondicionamento e transporte de cargas. 
Definição dos limites de preferência, indiferença e pesos dos critérios

Os pesos foram definidos por opinião de especialista, sendo que foram oferecidos a quatro especialistas os 11 critérios, para que cada um distribuísse 100 pontos de acordo com a importância de cada um. O peso adotado é a média aritmética simples da pontuação de cada critério dada por cada um dos quatro avaliadores e são apresentados na Tabela 1 , padronizados entre 0 e 1.

Tabela 1 - Ponderação dos critérios

\begin{tabular}{|c|c|c|c|c|c|c|c|c|c|c|c|}
\hline & Cr1 & Cr2 & $\mathrm{Cr} 3$ & Cr4 & $\mathrm{Cr} 5$ & Cr6 & Cr7 & Cr8 & $\mathrm{Cr} 9$ & Cr10 & Cr11 \\
\hline Peso & 0,1 & 0,2 & 0,3 & 0,3 & 0,5 & 0,5 & 0,4 & 0,4 & 0,8 & 0,3 & 0,3 \\
\hline
\end{tabular}

Fonte: Dados da pesquisa, 2007.

Todos os critérios têm sentido de preferência crescente, exceto o critério CR9, que possui sentido de preferência decrescente, isto é, quanto menor o valor do indicador, melhor é a avaliação.

Como são adotadas quatro classificações, são necessárias três bordas. A borda central é definida como a média aritmética com cortes de extremos (maior e menor valor) dos dados avaliados, e a borda superior e inferior são determinadas a um desvio-padrão (calculado também com cortes de extremos) de distância da borda central.

Tabela 2 - Limites de preferência, indiferença, e valores das bordas

\begin{tabular}{|c|c|c|c|c|c|c|c|c|c|c|c|}
\hline & Cr1 & $\mathrm{Cr} 2$ & Cr3 & $\mathrm{Cr} 4$ & Cr5 & Cr6 & Cr7 & $\mathrm{Cr} 9$ & Cr10 & Cr11 & Cr12 \\
\hline $\mathrm{p}$ & 0,1064 & 0,5109 & 1,04 & 0,6311 & 0,624 & 28,488 & 10,3583 & 37,008 & 1,696 & 1,568 & 197,022 \\
\hline$q$ & 0,053 & 0,255 & 0,52 & 0,315 & 0,612 & 14,488 & 5,14916 & 18,504 & 0,848 & 0,784 & 98,511 \\
\hline \multicolumn{12}{|c|}{ Ótimo } \\
\hline$b_{1}$ & 2,6576 & 11,65 & 15,96 & 14,77 & 11,23 & 631 & 201,27 & 700,06 & 33,37 & 33,98 & 4595 \\
\hline \multicolumn{12}{|c|}{ Bom } \\
\hline $\mathrm{b}_{2}$ & 1,33 & 6,387 & 13,001 & 7,888 & 7,8 & 362 & 129,47 & 462,6 & 21,2 & 19,6 & 2463 \\
\hline \multicolumn{12}{|c|}{ Regular } \\
\hline$b_{3}$ & 0,002 & 1,12 & 10,03 & 1,01 & 4,37 & 93 & 57,68 & 225,14 & 9,03 & 5,22 & 330 \\
\hline
\end{tabular}

Fonte: Dados da pesquisa, 2007.

Os limites de preferência (p) e indiferença (i) são determinados como sendo 8\% e $4 \%$ da média aritmética com cortes dos dados. 
Tabela 3 - Avaliações dos todos os portos à luz de todos os critérios

\begin{tabular}{l|rrrrrrrrrrr}
\hline \multicolumn{2}{r}{ Cr1 } & Cr2 & Cr3 & Cr4 & Cr5 & Cr6 & Cr7 & Cr8 & Cr9 & Cr10 & Cr11 \\
\hline A1 & 0,034 & 13 & 45 & 9 & 5 & 11 & 100 & 635 & 8 & 0 & 111 \\
A2 & 0,08 & 1,295 & 7,3 & 6 & 5 & 94 & 100 & 317 & 9 & 0 & 591 \\
A3 & 3,9203 & 8,6 & 20 & 1 & 6 & 49 & 100 & 218 & 9 & 0 & 247 \\
A4 & 0 & 1,5 & 12 & 5 & 8 & 202 & 100 & 200 & 10 & 4 & 686 \\
A5 & 135 & 0,58 & 15,5 & 14 & 13 & 61 & 90 & 234 & 11 & 4 & 2,806 \\
A6 & 0,047 & 0,8 & 12 & 9 & 11 & 629 & 100 & 282 & 21 & 18 & 2,343 \\
A7 & 0,15 & 5 & 10,5 & 5 & 16 & 314 & 97 & 634 & 28 & 19 & 3,104 \\
A8 & 7,7651 & 13,01 & 13,4 & 45 & 11 & 2,97 & 100 & 2816 & 134 & 53 & 27,387 \\
A9 & 0,036 & 14,97 & 13,11 & 4 & 4 & 386 & 100 & 499 & 27 & 36 & 2,801 \\
A10 & 0,135 & 4,7 & 10 & 1 & 4 & 774 & 100 & 962 & 41 & 40 & 6,249 \\
A11 & 0,4 & 0,3 & 10,5 & 7 & 4 & 39 & 100 & 303 & 17 & 6 & 256 \\
A12 & 0,75 & 9,8 & 13 & 25 & 11 & 1,074 & 100 & 542 & 39 & 16 & 5,544 \\
\hline
\end{tabular}

Fonte: Dados da pesquisa, 2007.

Resultados

A Tabela 4 apresenta três resultados distintos, o primeiro considerando a totalidade de indicadores $(\mathrm{T})$, o segundo somente os indicadores de infraestrutura (I), e o terceiro somente com os indicadores de desempenho (D).

Tabela 4 - Classificação dos portos sob a análise de todos os critérios

Todos os indicadores (T) Infraestrutura (I) Desempenho (D)

\begin{tabular}{l|lll}
\hline $\begin{array}{l}\text { Ótimo } \\
\text { Bom }\end{array}$ & A12 & - & - \\
Regular & A1, A2, A3, A4, A11 & A5, A12 & A6 \\
& & A7, A2, A3, A4, A6, A2, A11 & A2, A3, A4, A11 \\
Ruim & A5, A6, A7, A8, A9, A10 & - & A1, A5, A7, A8, \\
& & & A9, A10, A12 \\
\hline
\end{tabular}

Fonte: Dados da pesquisa, 2007.

Nenhum porto é classificado no estrato de otimalidade em nenhuma das três análises, sendo que porto de Rio Grande (A12) alcança a melhor classificação considerando todos os indicadores e em infraestrutura, mas lhe é atribuída a pior 
classificação quando considerados somente os indicadores de desempenho. É interessante notar que apesar de possuir uma boa infraestrutura, os resultados apontam para uma utilização deficiente desta.

Os portos de Belém (A2), Vila do Conde (A3), Fortaleza (A4) e Imbituba (A11) são classificados como regulares em todos os aspectos considerados, e a partir disto é possível afirmar que este conjunto de portos possui infraestrutura longe do ideal e também a utilizam de modo deficitário.

É interessante notar que nenhum porto foi alocado no pior estrado de infraestrutura, estando todos entre Bom (somente dois) e Regular (nove restantes). Ao confrontar este resultado com a coluna de desempenho, somente um (A6 Salvador) apresenta um bom desempenho, quatro apresentam um desempenho regular, e sete (grande maioria) apresentam um desempenho considerado ruim, corroborando com generalização de que o desempenho dos portos é pior que sua infraestrutura.

O conjunto de portos de Suape (A5), Vitória (A7), Santos (A8), São Francisco do Sul (A9) e Itajaí (A10) figura no pior estrato em Desempenho e na totalidade de indicadores, mas possuem uma avaliação tida como Regular quando considerada somente sua infraestrutura, com exceção do porto de Suape (A5), que possui uma boa infraestrutura, junto com o porto de Rio Grande (A12).

Analisando o desempenho nos critérios de infraestrutura dos portos brasileiros, pode-se observar que nenhum porto possui uma infraestrutura considerada ótima à luz de todos os critérios avaliados, mas, em compensação, não há nenhum com infraestrutura considerada ruim. A maioria é classificada como regular, sendo considerados bons os portos de Suape (A5) e de Rio Grande (A12), ambos possuindo capacidade instalada superior aos demais, segundo os resultados obtidos. Todos os demais são classificados no estrato de regular.

O porto de Manaus (A1) encontra-se apenas como regular, mesmo apresentando uma profundidade máxima excelente de $45 \mathrm{~m}$. Também o porto de Vila do Conde (A3), que apresenta dois critérios com excelente desempenho - Área Total (CR01) e Profundidade Máxima (CR03) -, mas que não foram suficientemente compensatórios para garantir uma boa classificação na análise. Assim como o porto de Santos (A8) que possui uma área total terrestre (CR01) grandiosa em relação aos demais portos, mas que também não foi o bastante para um bom desempenho coletivo.

\section{Conclusões}

O trabalho se ocupou de analisar criticamente uma classificação dos portos brasileiros que movimentam contêineres e realizou uma análise utilizando dois 
conjuntos de critérios: indicadores de desempenho de determinado ano e indicadores de infraestrutura, além de uma análise com todos os indicadores.

A questão da pesquisa que o trabalho se propôs a responder inquiria sobre a situação dos portos brasileiros. Adotou-se como hipótese que a situação é heterogênea, com alguns portos apresentando bom desempenho geral, e muitos com desempenho regular e ruim. Ao final do estudo de caso múltiplo, refuta-se parcialmente a hipótese declarada quando se verifica que a maioria dos portos foi classificada entre "Regular" e "Ruim", os estratos de piores performances, tanto em infraestrutura, quanto em desempenho.

Este trabalho revela uma estrutura deficitária aliada a um desempenho pífio dos portos, que pode ser considerado como um dos gargalos do desenvolvimento industrial via escoamento da produção destinada às exportações, prejudicando a logística de diversas cadeias de suprimento brasileiras e exteriores, que mesmo antes da crise de 2008, não estava adequada à realidade nacional.

A partir destes resultados e a conclusão obtidos neste trabalho, recomenda-se aos gestores investimentos localizados nas duas principais áreas abordadas neste estudo. Tanto na parte de infraestrutura, modernizando os portos e eliminando os gargalos de exportação e importação para o Brasil, e também em gestão, pois a infraestrutura atual não é utilizada, produzindo altos desempenhos.

Sobre o avanço metodológico, a definição dos limites utilizados para o funcionamento do algoritmo do ELECTRE TRI foi feita por método matemático a partir dos dados levantados, incorporando, de alguma forma, uma análise de benchmarking ao estudo. Esta experimentação de um método mais rígido, mas ainda assim com supervisão humana, caracteriza-se como uma contribuição à área da análise multicriterial. Os parâmetros adotados no método foram validados após observação de especialistas, mas são caracterizados também por serem advindos de um levantamento de dados públicos oficiais.

Por fim, abre-se como novo nicho a ser explorado, a constante avaliação da situação dos portos brasileiros, via de passagem das importações e exportações realizadas no território nacional, tanto dos portos de movimentação de contêineres, investigado neste trabalho, como os de outras modalidades, considerando suas peculiaridades. Sugere-se inclusive, a título de trabalho futuro, a replicação deste mesmo estudo de caso múltiplo, com os mesmos conjuntos de indicadores e conjunto de alternativas, em data futura, quando os efeitos da crise de 2008 já não se fizerem mais presentes na economia, para ser possível gerar resultados para comparação e avaliação de progresso ou não. 


\section{Referências}

ALMEIDA, A. T. Processo de decisão nas organizações: construindo modelos de decisão multicritério. So Paulo: Atlas, 2013.

ALMEIDA-DIAS, J.; FIGUEIRA, J. R.; ROY, B. Electre Tri-C: A multiple criteria sorting method based on characteristic reference actions. European Journal of Operational Research, v. 204, n. 3, p. 565-580, Agosto 2010.

ANTAQ. Desempenho Portuário. Disponível em: $<$ http://www.antaq.gov.br/portal/DesempenhoPortuario/Index.asp>. Acesso em: 11 jan. 2014.

Transportes Aquaviários, 2014. Disponível em: <http://www.antaq.gov.br/portal/pdf/BoletimPortuario/BoletimPortuarioPrime iroTrimestre2014.pdf>. Acesso em: 12 ago. 2014.

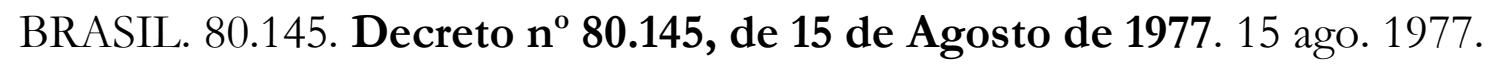

COSTA, H. G. et al. ELECTRE TRI aplicado a avaliação da satisfação de consumidores. Produção, v. 17, n. 2, p. 230-245, ago. 2007.

COSTA, H. G.; SOARES, A. C.; OlIVEIRA, P. F. DE. Avaliação de transportadoras de materiais perigosos utilizando o método ELECTRE TRI. Gestão \& Produção, v. 11, n. 2, p. 221-229, ago. 2004.

DEZERT, J.; TACNET, J.-M. Soft ELECTRE TRI outranking method based on belief functions. In: $15 \mathrm{TH}$ International Conference on Information Fusion, Singapore, 24 jul. 2012. Anais... Acesso em: 29 jul. 2012

Appendix to the paper entitled: Soft ELECTRE TRI outranking method based on belief functions. Technical appendix of a scientific paper. 2013.

DOUMPOS, M.; ZOPOUNIDIS, C. Multicriteria decision aid classification methods. Dordrecht: Kluwer Academic Publishers, 2002.

FIGUEIRA, J. R. M.; MOUSSEAU, V.; ROY, B. ELECTRE methods. In: FIGUEIRA, J. R. M.; GRECO, S.; EHRGOTT, M. (Eds.). Multiple criteria decision analysis: state of the art surveys. London: Kluwer Academic Publishers, 2005. p. 133-162. 
FREITAS, A. L. P. DE; COSTA, H. G. Ordenação e classificação de alternativas: uma análise multicritério. In: XX Encontro Nacional de Engenharia de Produção. Anais... São Paulo: ABEPRO, 2000.

- Uma análise multicritério para a classificação da qualidade de serviços utilizando o método ELECTRE TRI. In: XXIII Encontro Nacional de Engenharia de Produção. Anais... Ouro Preto: ABEPRO, 2003.

GIL, A. C. Como elaborar projetos de pesquisa. 4. ed. São Paulo: Atlas, 2007.

GOICOECHEA，A.; HANSEN，D. R.; DUCKSTEIN, L. Multiobjective decision analysis with engineering and business applications. Michigan: Wiley, 1982.

GOMES, L. F. A. M. Teoria da decisão. São Paulo: Thomson, 2007.

GOMES, L. F. A. M.; GOMES, C. F. S. Tomada de decisão gerencial: enfoque multicritério. 4. ed. São Paulo: Atlas, 2012.

GOMES, L. F. A. M.; GONZÁLEZ-ARAYA, M. C.; CARIGNANO, C. Tomada de decisões em cenários complexos: introdução aos modelos discretos de apoio multicritério à decisão. São Paulo: Thomson, 2004.

HORA, H. R. M. DA et al. Uma abordagem multicritério para classificação de softwares de matemática. In: XXVIII Encontro Nacional de Engenharia de Produção. Anais... Rio de Janeiro: ABEPRO, 2008. Disponível em: <http://www.es.iff.edu.br/softmat/download/leitu/Uma_abordagem_multicriter io.pdf $>$. Acesso em: 24 jan. 2013.

HORA, H. R. M. DA; MONTEIRO, G. T. R.; ARICA, J. Confiabilidade em questionários para qualidade: um estudo com o coeficiente Alfa de Cronbach. Produto \& Produção, v. 11, n. 2, p. 85-103, 24 jun. 2010.

KIRCHNER, L. H. C.; MEDEIROS, F. F. Movimentação de cargas nos portos brasileiros. Brasília: Agência Nacional de Transportes Aquaviários, jul. 2010. Disponível em: <http://www.antaq.gov.br/portal/pdf/BoletimPortuario/ BoletimPortuario2Trimestre2010.pdf>. Acesso em: 1 nov. 2014.

LACERDA, S. M. Investimentos nos portos brasileiros: Oportunidades da concessão da infra-estrutura portuária: Transportes. Rio de Janeiro: BNDES, 2005. Disponível em: <http://www.bndes.gov.br/SiteBNDES/export/ sites/default/bndes_pt/Galerias/Arquivos/conhecimento/bnset/set2209.pdf $>$. Acesso em: 12 ago. 2014. 
LIMA, E. C. P. DE; ALMEIDA, J. A. DE; ALMEIDA, A. T. DE. Modelo multicritério para seleção de projetos em uma empresa de serviços de consultoria. In: XXX Encontro Nacional de Engenharia de Produção. Anais... São Carlos: ABEPRO, 2010.

MONTEIRO, G. T. R.; HORA, H. R. M. Pesquisa em saúde pública: como desenvolver e validar instrumentos de coleta de dados. Curitiba: Appris, 2014.

MOREIRA, R. A. Análise Multicritério dos projetos do SEBRAE/RJ através do ELECTRE IV. Mestrado em Administração - Rio de Janeiro: Instituto Brasileiro de Mercado de Capitais, 2007.

MOTA, L. DE A. E. Capitalismo contemporâneo, desigualdades sociais e a crise de 2008. Revista Brasileira de Desenvolvimento Regional, v. 1, n. 1, p. 51-64, 1 mar. 2013.

OLIVEIRA, W. T. R. Utilizando integrais fuzzy em tomada de decisão multicritério. Mestrado em Ciências da Computação - Florianópolis: Universidade Federal de Santa Catarina (UFSC), 2003.

PARREIRAS, R. O. Algoritmos evolucionários e técnicas de tomada de decisão em análise multicritério. Doutorado em Engenharia Elétrica - Belo Horizonte: Universidade Federal de Minas Gerais (UFMG), 2006.

ROCHA, C. B.; MORATO, R. A. Gestão Portuária: análise comparativa entre modelos internacionais e propostas ao modelo atual brasileiro. In: Encontro Nacional da Associação Brasileira de Estudos Regionais e Urbanos (VII ENABER). Anais... São Paulo: 2009. Disponível em: <http://aplicativos.fipe. org.br/enaber/pdf/66.pdf>. Acesso em: 3 jan. 2014

ROY, B.; BOUYSSOU, D. Aide Multicritère à la décision: méthodes et cas. Paris: Economica, 1993.

SEP. Sistema Portuário Nacional. Disponível em: <http://www.portos dobrasil.gov.br/sistema-portuario-nacional>. Acesso em: 11 jan. 2014.

SILVA, E. L. S.; MENEZES, E. M. M. Metodologia da pesquisa e elaboração de dissertação. 4. ed. Florianópolis: UFSC, 2005.

SZAJUBOK, N. K.; MOTA, C. M. DE M.; ALMEIDA, A. T. DE. Uso do método multicritério ELECTRE TRI para classificação de estoques na construção civil. Pesquisa Operacional, v. 26, n. 3, p. 625-648, dez. 2006. 
Endereço para correspondência:

Angélica FariaCampanhão - angelicafcampanhao@yahoo.com.br Rua Antônio da Veiga, 140, Câmpus 1, Sala J-003

89012-900 Blumenau/SC, Brasil

Fernando Leste Nauck Fabiano Filho - fernandonauck@gmail.com Rua Antônio da Veiga, 140, Câmpus 1, Sala J-003 89012-900 Blumenau/SC, Brasil

Juliana Landim Gomes Siqueira - julianalandim_6@hotmail.com Rua Antônio da Veiga, 140, Câmpus 1, Sala J-003 89012-900 Blumenau/SC, Brasil

Rafaela Landim Gomes Siqueira - rafa_landim6@hotmail.com Rua Antônio da Veiga, 140, Câmpus 1, Sala J-003 89012-900 Blumenau/SC, Brasil

Henrique Rego Monteiro da Hora - dahora@gmail.com Av. Dário Vieira Borges, 235 28360-000 Bom Jesus do Itabapoana/RJ, Brasil

Helder Gomes Costa-hgc@pq.cnpq.br Av. Dário Vieira Borges, 235 28360-000 Bom Jesus do Itabapoana/RJ, Brasil 